\title{
C-Reactive Protein and Neutrophil to Lymphocyte Ratio Values in Predicting Inhospital Death in Patients with Stanford Type A Acute Aortic Dissection
}

\author{
Burak Erdolu, Ahmet Kağan As \\ Department of Cardiovasculer Surgery, University of Health Sciences, Bursa Yuksek Ihtisas Training and Research Hospital, \\ Yildırım/BURSA, Turkey
}

\section{ABSTRACT}

Background: Aortic dissection is a cardiovascular disease with high mortality and morbidity rates. The aim of this study is to investigate the role of C-reactive protein (CRP) and neutrophil-to-lymphocyte ratio (NLR) in predicting inhospital mortality in patients undergoing emergent surgery for Stanford type A aortic dissection.

Methods: Patients operated for acute Stanford type A aortic dissection between January 2010 and December 2018 were included in the study. Patients without in-hospital mortality were classified as Group 1, and patients with mortality were classified as Group 2.

Results: One-hundred-eighteen patients were involved in the study. Patient mean age was $57 \pm 11.7$ years, and 89 patients $(75.4 \%)$ were male. Neutrophil-to-lymphocyte ratio (NLR), white blood cell (WBC), neutrophil counts, and C-reactive protein (CRP) values at the time of admission also were found to be high in Group $2(P=.001, .021,<.001$, $<.001$ respectively). Total perfusion times (TPt), antegrade cerebral perfusion time (ACPt), cross-clamp time $(\mathrm{CCt})$, and intensive care unit (ICU) stay periods significantly were higher in the mortality group $(P<.001,<.001,=.01$, and $<.001$, respectively). In receiver-operating characteristic (ROC) curve analysis, a cut-off level of $23 \mathrm{mg} / \mathrm{L}$ was determined for CRP levels that predict progression to mortality (area under the curve (AUC): $0.879, P<.001,75.0 \%$ sensitivity and $58.0 \%$ specificity). Similarly, a cut-off level of 8.8 was found for NLR that predicts progression to mortality (AUC: $0.835, P<.001,76.0 \%$ sensitivity and $61.0 \%$ specificity).

Conclusion: As a result, we can use CRP and NLR values, which easily can be measured or calculated from blood tests to predict mortality in patients with aortic dissections, which may have serious mortal consequences.

\section{INTRODUCTION}

Aortic dissection (AD) is a cardiovascular disease with high mortality and morbidity rates. The estimated annual

Received May 10, 2020; accepted May 20, 2020.

Correspondence: Abmet Kağan As, MD, University of Health Sciences, Bursa Yuksek Ibtisas Training and Research Hospital, Department of Cardiovascular Surgery, Mimar Sinan Town. Emniyet Street. Yildirm/BURSA; +90224295-50-00; fax +90224-275-67-67 (e-mail: ahmetkagan_as@hotmail.com). prevalence of AD is 2.9-4.7 per 100,000 people [Pacini 2013]. Acute Type A aortic dissection (AAAD) is a pathology requiring urgent surgical intervention. Rupture of the intimal layer of the aorta causes AD. The complications may vary, depending on the end-organ involvement. Rupture of the aortic wall often is the cause of sudden death [Engin 2018; Huang 2018].

Inflammation plays an important role in the progress and prognosis of the AD. Inflammatory parameters, such as C-reactive protein (CRP) and white blood cells (WBC) can be useful in the prognosis of cardiovascular diseases, including aortic dissections [Luo 2009]. CRP is a non-specific acute phase reactant synthesized in the liver with induction of IL-6. This parameter, which can be measured quickly and easily, has an important role in the diagnosis of inflammatory diseases [Volanakis 2001]. That it may play a role in the prognosis of cardiovascular diseases, has been shown [Melander 2009].

Neutrophil-to-lymphocyte ratio (NLR) is known to play a role in the prognosis of many diseases. This ratio, which easily can be calculated from the complete blood count analysis, also can play a role in the prognosis of cardiovascular diseases

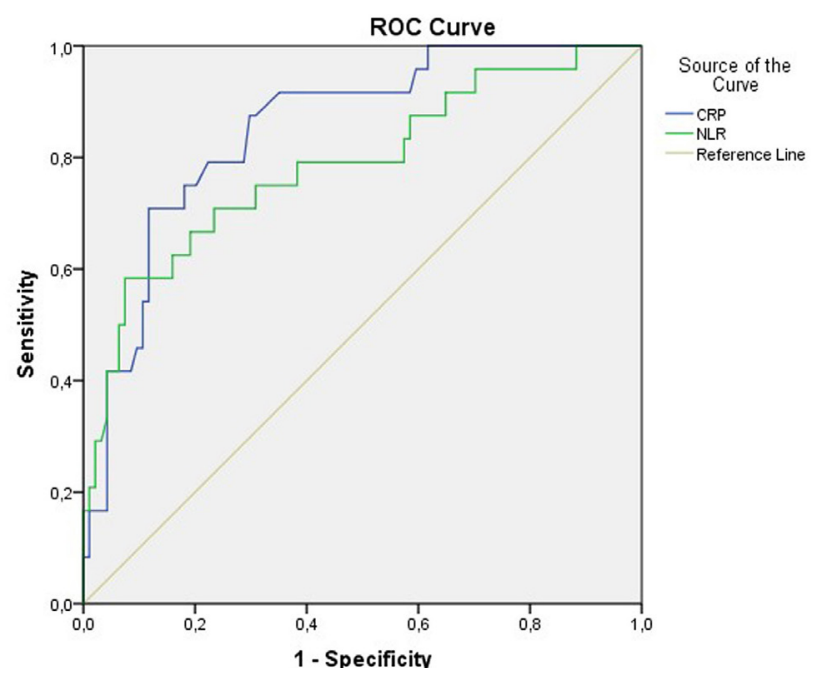

ROC curve and AUC (area under the curve) for CRP and NLR to predict mortality (CRP: cut-off: $23 \mathrm{mg} / \mathrm{L}$, AUC: $0.879,95 \% \mathrm{Cl}: 0.810-$ 0.949 , log rank $P<.001,75.0 \%$ sensitivity and $58.0 \%$ specificity. NLR cut-off level: 8.8 AUC: 0.835, 95\% Cl: 0.735-0.934, $\log \operatorname{rank} P<.001$, $76.0 \%$ sensitivity and $61.0 \%$ specificity). 
Table 1. Demographic features and blood values of the patients

\begin{tabular}{|c|c|c|c|}
\hline Age(years)(mean $\pm s d)$ & $57.2 \pm 12.3$ & $56.4 \pm 9.6$ & .742 \\
\hline Smoking, n (\%) & $52(55.3 \%)$ & $14(58.3 \%)$ & .791 \\
\hline Hypertension, n (\%) & $76(80.9 \%)$ & $21(87.5 \%)$ & .447 \\
\hline COPD, n (\%) & $13(13.8 \%)$ & $3(12.5 \%)$ & .865 \\
\hline Ejection fraction (\%)(mean $\pm s d)$ & $53.8 \pm 6.6$ & $51.2 \pm 6.9$ & .183 \\
\hline Hematocrit (\%)(mean \pm sd) & $42.9 \pm 4.8$ & $43.5 \pm 4.1$ & .701 \\
\hline $\mathrm{WBC}\left(10^{3} / \mathrm{mm}^{3}\right)(\operatorname{mean} \pm \mathrm{sd})$ & $13.3 \pm 2.8$ & $15.3 \pm 2.9$ & .001 \\
\hline Neutrophil, $10^{3} / \mathrm{ml}$, (mean $\pm \mathrm{sd}$ ) & $9.4 \pm 2.3$ & $10.4 \pm 2.1$ & .021 \\
\hline C-reactive protein $(\mathrm{mg} / \mathrm{L})(\operatorname{mean} \pm \mathrm{sd})$ & $15.5 \pm 7.3$ & $33.4 \pm 21.4$ & $<.001$ \\
\hline
\end{tabular}

COPD: Chronic obstructive pulmonary disease, WBC: White blood cells

[Karabinos 2009]. In this study, we aimed to determine the importance of CRP and NLR in predicting in-hospital mortality in patients undergoing emergent surgery for AAAD.

\section{PATIENTS AND METHODS}

Permission for the study was obtained from the local ethics committee. The patients operated for AAAD at the Bursa Yüksek İhtisas Education and Research Hospital, University of Health Sciences, between January 2010 and December 2018, were included in the study. Patients with active infection, known systemic inflammatory disease, traumatic dissection, a syndrome like Marfan Syndrome, with malperfusion or clinical shock at the time of admission, were excluded from the study.

After applying the exclusion criteria, 118 patients were involved in the study. The diagnosis of $\mathrm{AD}$ was confirmed by echocardiography and computerized tomography with contrast in all patients. Blood samples were collected before admission to the operation theatre. The patients were taken into operation theatre as soon as the dissection was diagnosed. All patients were operated with median sternotomy under general anesthesia. Antegrade cerebral perfusion with moderate hypothermia was used in all patients. Preoperative (age, sex, hypertension (HT), diabetes mellitus (DM), blood parameters, etc.), intraoperative (total perfusion time (TPt), cross-clamp time (CCt), antegrade cerebral perfusion time (ACPt), etc.), and postoperative (intensive care unit (ICU) stay, total hospital stay, etc.) data of the patients were recorded. Patients without in-hospital mortality were classified as Group 1, and patients with mortality were classified as Group 2. Predictive effects of CRP and NLR values measured at the time of admission on in-hospital mortality were investigated.
Laboratory measurements: Blood samples were taken from an arterial cannulation line of each patient in the intensive care unit. Hematologic parameters were measured by using an automated hematological analyzer (Coulter LH 780 Analyzer, CA, USA). In addition, NLR was calculated and blood parameters were recorded.

Statistical analysis: Statistical data was evaluated with the Statistical Package for the Social Sciences (IBM SPSS Statistic Inc. version 21.0, Chicago, IL). Continuous and ordinal variables were described as mean \pm standard deviation and nominal variables were described as frequency and percentage. Kolmogorov-Smirnov test and Shapiro-Wilk tests of normality were used to identify distribution of variables. Student's $t$ test was used to compare two groups for continuous variables with normal distribution. Mann-Whitney U test was used to compare two groups for continuous variables without normal distribution. Chi square test was used to compare two groups for nominal variables. Predictors of mortality were identified by using binary logistic regression analysis. Correlation analysis between CRP and NLR was performed using Spearman correlation test. For all tests, a $P$ value of $<.05$ was considered statistically significant. Receiveroperating characteristic (ROC) curve was performed for the prediction of mortality, and the area under the curve (AUC) was calculated for CRP and NLR.

\section{RESULTS}

One-hundred-eighteen patients were included in this study. Patient mean age was $57 \pm 11.7$ years, and 89 patients $(75.4 \%)$ were male. There were $97(82.2 \%)$ patients with 
Table 2. Intraoperative and postoperative features of the patients

\begin{tabular}{|c|c|c|c|}
\hline TPt (minutes) (mean \pm sd) & $144 \pm 39.1$ & $211.4 \pm 89.8$ & $<.001$ \\
\hline ACPt (minutes) (mean $\pm s d)$ & $29.4 \pm 15.4$ & $42.3 \pm 21.6$ & $<.001$ \\
\hline Concomitant CABG, n (\%) & $6(6.3 \%)$ & $2(8.3 \%)$ & .321 \\
\hline Total hospital stay (days) (mean \pm sd) & $9.3 \pm 2.1$ & $13.7 \pm 10.2$ & .051 \\
\hline
\end{tabular}

TPt: Total perfusion time, CCt: Cross-clamp time, ACPt: Antegrade cerebral perfusion time, CABG: coronary artery bypass graft, ICU: intensive care unit

hypertension, 17 with DM (14.4\%), 16 with chronic obstructive pulmonary disease (COPD) (13.5\%), and 66 smoking $(55.9 \%)$ patients. The mean ejection fraction (EF) was 53.3 \pm 6.7 . At the time of presentation, hematocrit values were 43 \pm 4.6, CRP values were $21.7 \pm 15.3, \mathrm{WBC}$ values were 13.7 \pm 2.9 , platelet values were $219.6 \pm 44.7$, and calculated NLR ratios were $8.6 \pm 1.7$. There was no significant effect of age, sex, EF, HT, smoking, DM, and COPD factors on mortality $(P>.05)$. Neutrophil-to-lymphocyte ratio, WBC, neutrophil counts and CRP values at the time of admission also were found to be high in Group $2(P=.001, .021,<.001,<.001$, respectively). The platelet and lymphocyte levels were similar in both groups $(P=.733, .052$, respectively) (Table 1$)$.

Eight $(6.7 \%)$ patients with coronary artery involvement underwent coronary bypass operation. In addition, there was no effect of coronary bypass operation on mortality $(P=.321)$. $\mathrm{TPt}, \mathrm{CCt}$, and ACPt values of the patients were $157.7 \pm 67.7$, $83.1 \pm 30.6$, and $32.1 \pm 17.5$, respectively. Duration of ICU stay was $5.6 \pm 6.3$ days and total hospitalization period was $10.2 \pm 5.2$ days. Total perfusion times, ACPt, CCt, and intensive care unit (ICU) stay periods significantly were higher in Group $2(P<.001,<.001,=.01$, and $<.001$, respectively $)$ (Table 2).

In hospital mortality was observed in 24 (20.3\%) patients. Factors related to the development of mortality were included univariate and multivariate logistic regression analysis. In unadjusted univariate logistic regression analysis, the development of mortality significantly was correlated with ICU stay (OR [odds ratio]: 1.614, 95\% CI [confidence interval]: 1.318-1.976, $P<.001)$, C-reactive protein (OR: 1.100, 95\% CI: 1.053-1.150, $P<.001)$, NLR (OR: 2.090, 95\% CI: 1.492-2.928, $P<.001)$, ACPt (OR: 1.036, 95\% CI: 1.010-1.062, $P=.005)$, TPt (OR: 1.018, 95\% CI: 1.008-1.028, $P<.001$ ), and CCt (OR: 1.017 , 95\% CI: $1.003-1.032, P=.020)$, but was not correlated with age and gender (Table 3). In addition, ICU stay, CRP, NLR, and TPt were identified as an independent predictor of development of mortality in multivariate analysis (OR: 2.700, 95\% CI: $1.382-5.273, P=.004$; OR: 1.151 , 95\% CI: $1.033-1.282$, $P=.011$; OR: $3.405,95 \%$ CI: $1.363-8.505, P=.009$ and OR: 1.021, 95\% CI: 1.004-1.039, $P=.018$, respectively) (Table 3 ).

In ROC curve analysis, for CRP it was determined a cutoff level of $23 \mathrm{mg} / \mathrm{L}$ for predicting progression of mortality
(AUC: $0.879,95 \%$ CI: $0.810-0.949, P<.001,75.0 \%$ sensitivity and $58.0 \%$ specificity), for NLR it was determined a cutoff level of 8.8 for predicting progression of mortality (AUC: 0.835, 95\% CI: $0.735-0.934, P<.001$, (76.0\% sensitivity and $61.0 \%$ specificity) (Figure). There was a moderate positive correlation between CRP and NLR $(\mathrm{R}=0.501, P<.001)$.

\section{DISCUSSION}

Aortic dissection, which may have serious mortal and morbid consequences, is a cardiovascular system disease that develops as a result of multiple factors, including genetics, atherosclerosis, and hypertension. The treatment of AAAD is always emergency surgery. Despite advances in technology, the desired mortality and morbidity results have not yet been achieved. One of the most important reasons for this is that intimal rupture may affect vital organ perfusion. Malperfusion at the time of the diagnosis decreases the chance of surgical success. Acute aortic dissections always have been interesting for cardiovascular surgeons. Several studies have been conducted on the factors affecting mortality. In these studies, advanced age, prolonged perfusion time, and prolonged intensive care stay were considered factors related with mortality. At the same time, it was determined that cardiac tamponade clinic and having a cerebrovascular disease is related with increased mortality rates [Cabasa 2016; Wu 2018].

Recent studies have shown that inflammatory markers may predict prognosis of various diseases. The effects of increased systemic inflammatory reactants on mortality in cardiovascular diseases have been indicated. At the time of presentation of $\mathrm{AD}$ patients, levels of inflammatory parameters in the blood can predict prognosis [Imtiaz 2012].

CRP levels rapidly increase in blood in systemic stress conditions; it is a sensitive marker of chronic vascular inflammation. Furthermore, atherosclerosis has some effects on vascular cell activation, apoptosis, monocyte accumulation, fat cell aggregation, and thrombosis stages [Yuan 2011]. CRP levels have been shown to affect disease-related complications and in-hospital mortality in AAAD [Komukai 2005; Eggebrecht 2004]. Sbarouni et al performed a study about CRP levels in three different groups comprising healthy 
Table 3. Logistic regression analysis for independent predictors to predict mortality

\begin{tabular}{|c|c|c|c|c|c|c|c|c|}
\hline Gender & .634 & .768 & .258 & 2.280 & - & - & - & \\
\hline ICU stay & $<.001$ & 1.614 & 1.318 & 1.976 & .004 & 2.700 & 1.382 & 5.273 \\
\hline NLR & $<.001$ & 2.090 & 1.492 & 2.928 & .009 & 3.405 & 1.363 & 8.505 \\
\hline $\mathrm{ACPt}$ & .005 & 1.036 & 1.010 & 1.062 & .532 & 1.020 & .959 & 1.085 \\
\hline $\mathrm{TPt}$ & $<.001$ & 1.018 & 1.008 & 1.028 & .018 & 1.021 & 1.004 & 1.039 \\
\hline $\mathrm{CCt}$ & .020 & 1.017 & 1.003 & 1.032 & .083 & .961 & .918 & 1.005 \\
\hline
\end{tabular}

ICU: intensive care unit, NLR: neutrophil-to-lymphocyte ratio, ACPt: antegrade cerebral perfusion time, TPt: total perfusion time, CCt: cross-clamp time

volunteers, patients with acute aortic dissection, and patients with aortic aneurysms. CRP levels significantly were higher in the two groups composed of patients than that of the healthy group [Sbarouni 2007]. Increased CRP levels have prognostic importance in acute aortic diseases. Schillinger et al conducted a study of patients with aortic dissection. They found that CRP levels were associated with in-hospital short-term adverse outcomes. In addition, they pointed out that CRP levels above $6.3 \mathrm{mg} / \mathrm{dl}$ were associated with poor prognosis [Schillinger 2002]. Furthermore, in a study by Sakakura et al., it was remarked that increased CRP values were related to poor prognosis and increased in-hospital mortality in acute aortic dissection cases [Sakakura 2010]. In our study, CRP levels at the time of admission significantly were higher in Group 2. We determined that CRP levels above $23 \mathrm{mg} / \mathrm{L}$ might affect in-hospital mortality. Therefore, it can be stated that the CRP levels measured at the time of admission would prefigure about the prognosis.

Prognostic value of NLR has been shown with many studies in many fields of medicine [Ay 2014; Oz 2017; Erdolu 2020]. Neutrophils can cause extracellular matrix and vascular smooth muscle damage in the aneurysmatic vessel wall [Folkesson 2007]. It can be told that this situation leads up to aortic dissection. Kalkan et al performed a study in patients with aortic dissection. In this study, the patients were divided into two groups as NLR $>6$ and NLR $<6$. The rates of mortality, major bleeding, and hospital-acquired infections were found significantly higher in the group of patients with NLR above 6 [Kalkan 2017]. In another study by Lafci et al, high NLR was found to be associated with increased dissection mortality. In this study, increased perfusion times, platelet counts, surgical procedures, and increased intensive care stay were found to be the factors affecting mortality. The cut-off value for NLR was 8 in the ROC analysis (sensitivity was $70 \%$; specificity was $53 \%$ ) [Lafci 2014].

Karakoyun et al investigated the relationship between the mortality and NLR in patients with acute aortic dissection. They stated that NLR $>8.5$ was associated with mortality. They also found significantly moderate positive correlation between CRP and NLR $(\mathrm{R}=0.506, P=.002)$ [Karakoyun 2015]. Similarly, in our study, there was a moderate positive correlation between CRP and NLR. Hypertension is one of the most important factors in the progress of the dissection. Possibly, the frequent occurrence of hypertension in patients with dissection may be the reason of increased NLR. This inflammatory process also may cause postoperative poor outcomes in operated patients [Tian 2010].

In our study, we aimed to determine the predictive values of CRP and NLR, known as inflammatory biomarkers, on mortality of the patients who underwent emergent operation for AAAD. Preoperative CRP and NLR values were found to be significantly higher in the group of patients with mortality. At the same time, prolonged total perfusion time and prolonged ICU stay were evaluated as independent predictors of mortality.

Our results may have been affected by our treatment methods. The relatively small number of patients also is a limitation of the study. The surgical interventions were performed by different surgeons, making standardization difficult. Further prospective studies are needed with a large patient series.

\section{CONCLUSION}

As a result, we can use CRP and NLR values, which easily can be measured or calculated from blood tests to predict mortality in patients with aortic dissections, which may have serious mortal consequences. In this field, we believe that more valuable results can be achieved with more multi-center prospective data-based studies with large patient groups.

\section{REFERENCES}

Ay D, Erdolu B, Yumun G, Aydin U, Demir A, Tiryakioglu O, et al. 2014. Comparing the effectiveness of neutrophil-lymphocyte ratio as a mortality predictor on middle and advanced age coronary artery bypass graft patients. North Clin Istanb 1(2): 95-100. 
Cabasa A, Pochettino A. 2016. Surgical management and outcomes of type A dissection-the Mayo Clinic experience. Ann Cardiothorac Surg 5(4):296-309.

Eggebrecht H, Naber CK, Bruch C, Kroger K, von Birgelen C, Schmermund A, et al. 2004. Value of plasma fibrin D-dimers for detection of acute aortic dissection. J Am Coll Cardiol 44: 804-9.

Engin M, Goncu MT, Guvenc O, Savran M, Ozyazicioglu AF. 2018. Retrospective Investigation of Factors Affecting Early Period Mortality And Morbidity After Operation in Type A Aortic Dissections. Dicle Med J 45 (4):387-396.

Erdolu B, As AK, Engin M. 2020. The Relationship between the HATCH Score, Neutrophil to Lymphocyte Ratio and Postoperative Atrial Fibrillation Afer O -Pump Coronary Artery Bypass Graf Surgery. Heart Surg Forum 23(1):E88-E92.

Folkesson M, Kazil M, Zhu C, Silveria A, Hemdahl AL, Hamsten A, et al. 2007. Presence of NGAL/ MMP-9 complexes in human abdominal aortic aneurysms. Thromb Haemost 98(02): 427-433.

Huang Q, Ren Y, Li H, et al. 2018. Systems Biology Approaches-based Biomarkers Discovery for Acute Aortic Dissection. Heart Surg Forum 21(5):E365-E369.

Imtiaz F, Shafique K, Mirza SS, Ayoob Z, Vart P, Rao S. 2012. Neutrophil lymphocyte ratio as a measure of systemic inflammation in prevalent chronic diseases in Asian population. Int Arch Med 5:2.

Kalkan ME, Kalkan AK, Gundes A, Yanartas M, Ozturk S, Gurbuz AS, et al. 2017. Neutrophil to lymphocyte ratio: a novel marker for predicting hospital mortality of patients with acute type A aortic dissection. Perfusion 32(4): 1-7.

Karabinos I, Koulouris S, Kranidis A, Pastromas S, Exadaktylos N, Kalofoutis A. 2009. Neutrophil count on admission predicts major in-hospital events in patients with a non-ST-segment elevation acute coronary syndrome. Clin Cardiol 32: 561-568.

Karakoyun S, Gürsoy MO, Akgün T, Öcal L, Kalçık M, Yesin M, et al. 2015. Neutrophil-lymphocyte ratio may predict in-hospital mortality in patients with acute type A aortic dissection. Herz 40(4):716-21.

Komukai K, Shibata T, Mochizuki S. 2005. C-reactive protein is related to impaired oxygenation in patients with acute aortic dissection. Int Heart J 46: 795-9.
Lafcı G, Cicek OF, Uzun HA, Yalcınkaya A, Diken AI, Turak O, et al. 2014. Relationship of admission neutrophil-to-lymphocyte ratio with inhospital mortality in patients with acute type I aortic dissection. Turk J Med Sci 44: 186-192.

Luo F, Zhou XL, Li JJ, Hui RT. 2009. Inflammatory response is associated with aortic dissection. Ageing Res Rev 8: 31-35.

Melander O, Newton-Cheh C, Almgren P, Hedblad B, Berglund G, Engstrom G, et al. 2009. Novel and conventional biomarkers for prediction of incident cardiovascular events in the community. JAMA 302:49-57.

Oz K, Iyigun T, Karaman Z, et al. 2017. Prognostic Value of Neutrophil to Lymphocyte Ratio and Risk Factors for Mortality in Patients with Stanford Type A Aortic Dissection. Heart Surg Forum 20(3):E119-E123.

Pacini D, Di Marco L, Fortuna D, Beatrice Belotti LM, Gabbieri D, Zussa C, et al. 2013. Acute aortic dissection: epidemiology and outcomes. Int J Cardiol 167: 2806-2812.

Sakakura K, Kubo N, Ako J, Wada H, Fujiwara N, Funayama H, et al. 2010. Peak C-reactive protein level predicts long-term outcomes in type $\mathrm{B}$ acute aortic dissection. Hypertension 55: 422-429.

Sbarouni E, Georgiadou P, Marathias A, Geroulanos S, Kremastinos DT. 2007. D-dimer and BNP levels in acute aortic dissection. International Journal of Cardiology 122:170-172.

Schillinger M, Domanovits H, Bayegan K et al. 2002. C-reactive protein and mortality in patients with acute aortic disease. Intensive Care Med 28:740-745

Tian N, Penman AD, Mawson AR et al. 2010. Association between circulating specific leukocyte types and blood pressure: the atherosclerosis risk in communities (ARIC) study. J Am Soc Hypertens 4:272-283.

Volanakis JE. 2001. Human C-reactive protein: expression, structure, and function. Mol Immunol 38: 189-197.

Wu Y, Jiang R, Xu P, et al. 2018. Perioperative Results and Risk Factors for In-Hospital Mortality In Patients With Stanford Type A Aortic Dissection Undergoing Sun's Procedure - A Single Center Study. Heart Surg Forum 21(5):E432-E437.

Yuan SM, Shi YH, Wang JJ, Lu FQ, Gao S. 2011. Elevated plasma $\mathrm{D}$-dimer and hypersensitive Creactive protein levels may indicate aortic disorders. Braz J Cardiovasc Surg 26(4):573-81. 